\begin{tabular}{|c|c|}
\hline Title & Derivation of the generalized Langevin equation in nonstationary environments \\
\hline Author(s) & Kawai, Shinnosuke; Komatsuzaki, Tamiki \\
\hline Citation & $\begin{array}{l}\text { Journal of Chemical Physics, 134(11), } 114523 \\
\text { https://doi.org/10.1063/1.3561065 }\end{array}$ \\
\hline Issue Date & 2011-03-21 \\
\hline Doc URL & http:/hdl.handle.net/2115/45147 \\
\hline Rights & $\begin{array}{l}\text { Copyright } 2011 \text { A merican Institute of Physics. This article may be downloaded for personal use only. A ny other use } \\
\text { requires prior permission of the author and the A merican Institute of Physics. The following article appeared in J. } \\
\text { Chem. Phys. 134, 114523 (2011) and may be found at https://dx.doi.org/10.1063/1.3561065 }\end{array}$ \\
\hline Type & article \\
\hline File Information & JCP13411_114523.pdf \\
\hline
\end{tabular}

Instructions for use 


\title{
Derivation of the generalized Langevin equation in nonstationary environments
}

\author{
Shinnosuke Kawai ${ }^{\mathrm{a})}$ and Tamiki Komatsuzaki \\ Molecule \& Life Nonlinear Sciences Laboratory, Research Institute for Electronic Science, Hokkaido \\ University, Kita 20 Nishi 10, Kita-ku, Sapporo 001-0020, Japan
}

(Received 28 December 2010; accepted 11 February 2011; published online 18 March 2011)

\begin{abstract}
The generalized Langevin equation (GLE) is extended to the case of nonstationary bath. The derivation starts with the Hamiltonian equation of motion of the total system including the bath, without any assumption on the form of Hamiltonian or the distribution of the initial condition. Then the projection operator formulation is utilized to obtain a low-dimensional description of the system dynamics surrounded by the nonstationary bath modes. In contrast to the ordinary GLE, the mean force becomes a time-dependent function of the position and the velocity of the system. The friction kernel is found to depend on both the past and the current times, in contrast to the stationary case where it only depends on their difference. The fluctuation-dissipation theorem, which relates the statistical property of the random force to the friction kernel, is also derived for general nonstationary cases. The resulting equation of motion is as simple as the ordinary GLE, and is expected to give a powerful framework to analyze the dynamics of the system surrounded by a nonstationary bath. (C) 2011 American Institute of Physics. [doi:10.1063/1.3561065]
\end{abstract}

\section{INTRODUCTION}

Many chemical events such as chemical reactions involve a huge number of atoms. For the understanding of the complex manybody behavior, one has represented the behavior by projecting the total system onto some selected degrees of freedom (dof) with friction kernel and random force arising from all the other dof called bath. ${ }^{1,2}$ The equation of motion for the selected variables is called generalized Langevin equation (GLE). ${ }^{3}$ While there is a proof ${ }^{3-5}$ that any dynamical system can be reduced to the form of GLE, it is useful only when we know, or can reasonably assume, the statistics of the random force appearing as a stochastic variable in GLE. Thus an equilibrium distribution of the bath is usually assumed when the GLE is used. ${ }^{3}$ An example of such statistical property of the random force is the fluctuation-dissipation theorem, where the autocorrelation function of the random force is related to the friction kernel. It was found recently ${ }^{6-17}$ that even though one cannot know an instantaneous value of the random force in advance since the initial condition of the bath is unknown, the statistical property enables us to analytically derive the boundary of the reaction in the state space, that is, a surface on which the system should end up with the reactant and the product with equal probability of one half. Following the pioneering works by Kramers ${ }^{1}$ and by Grote and Hynes, ${ }^{2}$ great progress $^{6-21}$ in the study of reaction dynamics in condensed phase have been made by using the GLE or the Langevin equation (a memoryless limit of GLE).

The statistical property of the random force is derived by assuming a thermal equilibrium (or more generally, a stationary distribution) of the bath dof. More precisely, the distribution is assumed to be a so-called constrained equilibrium, ${ }^{3}$

\footnotetext{
a)Electronic mail: skawai@es.hokudai.ac.jp. Research Fellow of the Japan Society for the Promotion of Science.
}

where the initial condition of the system can be specified arbitrarily but the distribution of the other modes (bath) is in equilibrium. However, one has often encountered many nonequilibrium molecular phenomena occurring with a nonstationary initial distribution of the bath. For example, in photo-excited reactions the initial distribution is determined by the response of the system to the light and thus can be different from the stationary one even along the coordinate other than the naïve "reaction coordinate." Sometimes one also excites the bath mode vibrations to obtain different reaction product. ${ }^{22-24}$ How we can acquire a low-dimensional description for such nonstationary systems has been a contemporary intriguing subjects to be resolved.

For the last decade there have been several attempts at establishing the nonstationary representation termed as the irreversible generalized Langevin equation (iGLE) ${ }^{25-34}$ In particular, the idea of generalized friction kernel ${ }^{28,34}$ dependent on both the time values at the past and the present was proposed. As the (nonstationary) bath relaxes to the equilibrium state as time proceeds, the friction kernel is shown to converge to the usual equilibrium one, which depends only on the time difference. The generalization of the fluctuation-dissipation theorem was also proposed: the autocorrelation of the random force averaged over a given distribution of the initial condition of the bath dof corresponds to the generalized time-dependent friction kernel $\mathrm{l}^{28-34}$ or with an exponentially dumping correction term, ${ }^{25-27}$ depending on how to formulate the iGLE. The iGLE can be applied not only to phenomena subject to an outside force that changes the solvent response, ${ }^{28}$ but also to systems whose nonstationarity is induced by the dynamics of the system itself. ${ }^{30}$ The growth of polymer was taken as an example where the property of the environment changes with the increase of the polymer length. It can also be applied to temperature-ramped chemical reactions ${ }^{31}$ where the 
environment temperature changes with time due to heating, for example, by a laser pulse. A further extension of iGLE (Ref. 32) was provided to describe an ensemble of systems each of which is subject to a different random force and which are allowed to interact with each other.

Despite their stimulating insights, these theories, however, have been so far limited to special systems where the bath is assumed to be a collection of harmonic oscillators and their coupling to the system is bilinear. Especially, they require a special form for the total-system Hamiltonian that depends on the history of the system dynamics, ${ }^{29,33}$ in deriving the form of iGLE in a heuristic fashion. ${ }^{28}$

In this paper, we present a rigorous and general derivation of iGLE that does not depend on any specific form of the original Hamiltonian, by using the projection-operator formalism. $^{3-5}$ With the firm mathematical framework free from the setting of the form of the underlying Hamiltonian, the iGLE is expected to be the potential means to address various dynamical events occurring in nonequilibrium, fluctuating environments. This paper is organized as follows: after reviewing the GLE and the iGLE in Sec. II, we present a theory for generalizing them to any nonstationary environment in Sec. III. The versatility of the theory is demonstrated numerically by using a simple example in Sec. IV. Finally, in Sec. V, the summary and outlook is presented.

\section{REVIEW OF GLE AND IGLE} by $^{3,5}$

The most generic form of the nonlinear GLE is given $\frac{\mathrm{d}^{2}}{\mathrm{~d} t^{2}} q_{j}=-\frac{\partial V(\boldsymbol{q})}{\partial q_{j}}-\int_{0}^{t} K_{j}\left(t-t^{\prime} ; \boldsymbol{q}\left(t^{\prime}\right), \dot{\boldsymbol{q}}\left(t^{\prime}\right)\right) \mathrm{d} t^{\prime}+\xi_{j}(t)$,

where $\boldsymbol{q}=\left(q_{1}, q_{2}, \ldots, q_{n}\right)$ is a set of variables to describe the system, and $V(\boldsymbol{q})$ is the potential of mean force, whose gradient with respect to $\boldsymbol{q}$ gives the force felt by the system averaged over the probability distribution of the bath. The friction term $K_{j}\left(t-t^{\prime} ; \boldsymbol{q}\left(t^{\prime}\right), \dot{\boldsymbol{q}}\left(t^{\prime}\right)\right)$ depends on the history of the system. Possible physical interpretation of this term is that the bath is kicked by the system at time $t^{\prime}$ and this kick affects the bath configuration and its dynamics after time $t^{\prime}$. Then, at time $t$, the system feels a force from the bath that depends on the bath configuration at time $t$, the latter in turn depending on the kick in the past (at time $t^{\prime}$ ) by the system. If the bath is in a stationary distribution, this response of the bath only depends on the time difference, hence we have the argument $t-t^{\prime}$ in $K$. The time-dependent force $\xi_{j}(t)$ represents the force from the bath that is purely determined by the initial condition of the bath. This third term is the so-called random force, because we only observe the system and do not know the initial condition of the bath.

One may expand the friction term in polynomial form

$K_{j}\left(t-t^{\prime} ; \boldsymbol{q}\left(t^{\prime}\right), \dot{\boldsymbol{q}}\left(t^{\prime}\right)\right)=\sum_{\boldsymbol{n}, \boldsymbol{m}} \gamma_{\boldsymbol{n} \boldsymbol{m}}\left(t-t^{\prime}\right) \boldsymbol{q}\left(t^{\prime}\right)^{\boldsymbol{m}} \dot{\boldsymbol{q}}\left(t^{\prime}\right)^{\boldsymbol{n}}$, or, more generally, in a complete set of basis functions $\left\{\phi_{n}\right\}$ :

$$
K_{j}\left(t-t^{\prime} ; \boldsymbol{q}\left(t^{\prime}\right), \dot{\boldsymbol{q}}\left(t^{\prime}\right)\right)=\sum_{n} \gamma_{n}\left(t-t^{\prime}\right) \phi_{n}\left(\boldsymbol{q}\left(t^{\prime}\right), \dot{\boldsymbol{q}}\left(t^{\prime}\right)\right) .
$$

Approximation of $K_{j}$ by truncating the summation and taking only the terms proportional to the velocity in these expansions yields

$$
K_{j}\left(t-t^{\prime} ; \boldsymbol{q}\left(t^{\prime}\right), \dot{\boldsymbol{q}}\left(t^{\prime}\right)\right) \approx \sum_{i} \gamma_{i j}\left(t-t^{\prime}\right) \dot{q}_{i}\left(t^{\prime}\right),
$$

giving the approximated GLE

$$
\frac{\mathrm{d}^{2}}{\mathrm{~d} t^{2}} q_{j}=-\frac{\partial V(\boldsymbol{q})}{\partial q_{j}}-\int_{0}^{t} \sum_{i} \gamma_{i j}\left(t-t^{\prime}\right) \dot{q}_{i}\left(t^{\prime}\right) \mathrm{d} t^{\prime}+\xi_{j}(t) .
$$

This is the most commonly used form of the GLE. This is shown ${ }^{35}$ to be exact, when the bath is a collection of harmonic oscillators and the coupling to the system is bilinear. The friction kernel and the random force are related by the fluctuation-dissipation theorem:

$$
\left\langle\xi_{i}(t) \xi_{j}\left(t^{\prime}\right)\right\rangle=k_{\mathrm{B}} T \gamma_{i j}\left(t-t^{\prime}\right),
$$

where the bracket denotes the ensemble average over stationary distribution, $k_{\mathrm{B}}$ is the Boltzmann constant, and $T$ is the temperature.

For stationary state, the friction kernel $\gamma_{i j}$ depends only on the time difference. References 28-33, and 34 proposed the extension of Eq. (5) to the nonstationary case as

$$
\frac{\mathrm{d}^{2}}{\mathrm{~d} t^{2}} q_{j}=-\frac{\partial V(\boldsymbol{q})}{\partial q_{j}}-\int_{0}^{t} \sum_{i} \gamma_{i j}\left(t, t^{\prime}\right) \dot{q}_{i}\left(t^{\prime}\right) \mathrm{d} t^{\prime}+\xi_{j}(t),
$$

and called it iGLE. The key difference is the dependence of the friction kernel $\gamma_{i j}\left(t, t^{\prime}\right)$ on both the "initial" $\left(t^{\prime}\right)$ and the "final" $(t)$ times. They further proposed a scaling of the random force as

$$
\xi_{j}(t)=g(t) \xi_{j}^{\mathrm{eq}}(t)
$$

where the superscript "eq" denotes the equilibrium state, and $g(t)$ is the scaling factor depending on time $t$. Extension of the fluctuation-dissipation theorem was also proposed,

$$
\left\langle\xi_{i}(t) \xi_{j}\left(t^{\prime}\right)\right\rangle=k_{\mathrm{B}} T \gamma_{i j}\left(t, t^{\prime}\right),
$$

where $\langle\cdot\rangle$ is an ensemble average with respect to a given distribution of the bath. Combining Eqs. (8) and (9) together with the fluctuation-dissipation relation at the equilibrium yields

$$
\gamma_{i j}\left(t, t^{\prime}\right)=g(t) \gamma_{i j}^{\mathrm{eq}}\left(t-t^{\prime}\right) g\left(t^{\prime}\right)
$$

where $\gamma_{i j}^{\text {eq }}$ is the equilibrium friction kernel. Reference 34 introduced a more generalized form of the friction kernel allowing for multiple heat baths and time dilatation in the arguments of the equilibrium friction kernels:

$$
\gamma_{i j}\left(t, t^{\prime}\right)=\sum_{k} \frac{T_{k}(t)}{T_{k}(0)} g_{k}(t) g_{k}\left(t^{\prime}\right) \gamma_{i j}^{\mathrm{eq}(k)}\left(\tau_{k}(t)-\tau_{k}\left(t^{\prime}\right)\right),
$$


where $k$ labels each heat bath and $T_{k}$ is the time-dependent "temperature" of the $k$ th bath. The function $\tau_{k}(t)$ is a monotonically increasing function of $t$ with time-dependent rate of increase reflecting the change of the frequency (and therefore the characteristic response time) of each bath with time.

In order to obtain Eqs. (7)-(11), however, they needed to assume a special type of the total Hamiltonian where the bath is harmonic, the coupling is bilinear, and there is a potential depending on the history, ${ }^{29}$ which was called nonlocal potential. The equivalence between this nonlocal Hamiltonian and iGLE was proved theoretically in Ref. 29 and its numerical evidence was provided in Ref. 33 within the framework of bilinearly coupled harmonic oscillators Hamiltonian systems.

In this paper, we present a general derivation of iGLE without imposing any particular form of the total Hamiltonian. The summary of the results in this article is:

- The idea of the time-dependent friction kernel [Eq. (7)] holds generally without any assumption about the total system.

- In contrast to the previous simplified treatments, the mean force potential $V(\boldsymbol{q})$ in Eq. (7) must be time dependent in general nonstationary cases.

- The extended fluctuation-dissipation theorem [Eq. (9)] holds generally for any irreversible system with an appropriate reinterpretation of the "temperature."

- The simple scaling [Eqs. (8) and (10)] does not hold for general nonstationary cases.

\section{GENERAL DERIVATION}

\section{A. Settings}

Let the phase space of the total system (including both the system and the bath) be

$$
\mathcal{N}=\{(\boldsymbol{q}, \boldsymbol{p})\},
$$

where $\boldsymbol{q}=\left(q_{1}, q_{2}, \ldots, q_{N}\right)$ denotes the position coordinates of the total system and $\boldsymbol{p}$ their conjugate momenta. The equation of motion is given by

$$
\begin{aligned}
\frac{\mathrm{d}}{\mathrm{d} t} q_{j} & =\frac{\partial H}{\partial p_{j}}, \\
\frac{\mathrm{d}}{\mathrm{d} t} p_{j} & =-\frac{\partial H}{\partial q_{j}},
\end{aligned}
$$

with the Hamiltonian $H$ which is a (possibly time-dependent) function on $\mathcal{N}$ :

$$
H=H(\boldsymbol{q}, \boldsymbol{p}, t) .
$$

We define the Liouvillian operator i $\hat{\mathscr{L}}$ by

$$
\mathrm{i} \hat{\mathscr{L}} A \stackrel{\text { def }}{=}\{A, H\}=\sum_{j}\left(\frac{\partial A}{\partial q_{j}} \frac{\partial H}{\partial p_{j}}-\frac{\partial A}{\partial p_{j}} \frac{\partial H}{\partial q_{j}}\right),
$$

for any function $A$ on $\mathcal{N}$ (complex-valued in general),

$$
A: \mathcal{N} \rightarrow \mathbb{C},
$$

and $\{\cdot, \cdot\}$ denotes the Poisson bracket.
Let us consider a distribution $\rho(\boldsymbol{q}, \boldsymbol{p}, t)$ on the phase space $\mathcal{N}$. The time evolution of the distribution is given by the Liouville equation

$$
\frac{\partial \rho}{\partial t}+\{\rho, H\}=0
$$

that is,

$$
\frac{\partial \rho}{\partial t}=-i \hat{\mathscr{L}} \rho
$$

The distribution is called stationary if $\partial \rho / \partial t=0$, and nonstationary otherwise. The word equilibrium distribution refers to either the canonical

$$
\rho \propto \exp \left(-\frac{H}{k_{\mathrm{B}} T}\right),
$$

or the microcanonical distribution

$$
\rho \propto \delta(H-E)
$$

where $\delta(\cdot)$ denotes Dirac's delta function. It is an easy matter to prove that the equilibrium distribution is stationary, but not all stationary distributions are equilibrium.

The time dependence of the distribution, and the possible time dependence of the Hamiltonian, can be treated similarly to the stationary case when we change to the extended phase space, ${ }^{36}$

$$
\mathcal{M} \stackrel{\text { def }}{=}\left\{\left(\boldsymbol{q}, \boldsymbol{p}, t, P_{t}\right)\right\},
$$

where the time $t$ is treated as a dynamical variable, and $P_{t}$ is the conjugate momentum to $t$ taking the same value as $-H$. A new independent variable $\tau$ is used to describe the evolution of the trajectory by the equation of motion,

$$
\begin{aligned}
\frac{\mathrm{d}}{\mathrm{d} \tau} q_{j} & =\frac{\partial K}{\partial p_{j}}=\frac{\partial H}{\partial p_{j}} \\
\frac{\mathrm{d}}{\mathrm{d} \tau} p_{j} & =-\frac{\partial K}{\partial q_{j}}=-\frac{\partial H}{\partial q_{j}}, \\
\frac{\mathrm{d}}{\mathrm{d} \tau} t & =\frac{\partial K}{\partial P_{t}}=1, \\
\frac{\mathrm{d}}{\mathrm{d} \tau} P_{t} & =-\frac{\partial K}{\partial t},
\end{aligned}
$$

with the extended Hamiltonian $K$ defined by

$$
K\left(\boldsymbol{q}, \boldsymbol{p}, t, P_{t}\right) \stackrel{\text { def }}{=} H(\boldsymbol{q}, \boldsymbol{p}, t)+P_{t} .
$$

Note that $\mathrm{d} t / \mathrm{d} \tau=1$, therefore by setting the initial condition $\left.t\right|_{\tau=0}=0$ we have

$$
t=\tau,
$$

and the same equation of motion for $(\boldsymbol{q}, \boldsymbol{p})$ with Eq. (13) is obtained.

We define the extended Liouvillian operator i $\hat{\Lambda}$ by

$$
\mathrm{i} \hat{\Lambda} F \stackrel{\text { def. }}{=} \hat{\mathscr{L}} F+\frac{\partial F}{\partial t},
$$

for any differentiable function $F$ on $\mathcal{M}$. The time evolution of a function $F=F(\boldsymbol{q}, \boldsymbol{p}, t)$ is given by

$$
F(\tau)=F(\tau ; \boldsymbol{q}, \boldsymbol{p}, t) \stackrel{\text { def }}{=} \exp (\mathrm{i} \hat{\Lambda} \tau) F,
$$


where we use the symbol $F(\tau)$ as an abbreviation of $F(\tau ; \boldsymbol{q}, \boldsymbol{p}, t)$. Note that, although we use the same symbol $F$ for the original function $F(\boldsymbol{q}, \boldsymbol{p}, t)$ and its time evolution $F(\tau ; \boldsymbol{q}, \boldsymbol{p}, t)$, their functional forms are different (distinguished by the notation of the argument " $\tau$;"). It is because the operation of $\exp (\mathrm{i} \hat{\Lambda}(\boldsymbol{q}, \boldsymbol{p}, t) \tau)$ on $F(\boldsymbol{q}, \boldsymbol{p}, t)$ in Eq. (26) results in a complicated, nonlinear function of $\boldsymbol{q}, \boldsymbol{p}$, and $t$, whose functional form becomes different from the original $F$. It is known that the function $F(\tau ; \boldsymbol{q}, \boldsymbol{p}, t)$ gives the value of the physical quantity $F$ at $\tau$ as a function of the initial condition $(\boldsymbol{q}, \boldsymbol{p}, t)$ at $\tau=0$ :

$$
F(\tau ; \boldsymbol{q}(0), \boldsymbol{p}(0), t(0))=F(\boldsymbol{q}(\tau), \boldsymbol{p}(\tau), t(\tau)),
$$

where $(\boldsymbol{q}(\tau), \boldsymbol{p}(\tau), t(\tau))$ is a trajectory evolving by the equation of motion Eq. (22). Equation (27) can be proved by Eqs. (22), (25) and (26) (e.g., see the appendix on the Lie transformation in Ref. 37).

The time-dependent distribution $\rho(\boldsymbol{q}, \boldsymbol{p}, t)$ can be regarded as a "distribution" on the extended phase space $\mathcal{M}$. From Eqs. (18) and (25) it is seen that

$$
\mathrm{i} \hat{\Lambda} \rho(\boldsymbol{q}, \boldsymbol{p}, t)=0 .
$$

In this sense we can regard $\rho(\boldsymbol{q}, \boldsymbol{p}, t)$ as a "stationary distribution" in the extended phase space $\mathcal{M}$. By virtue of Eq. (28), we can follow a mathematical procedure similar to that of the projection operator formalism as was done for the stationary system. ${ }^{3-5}$

\section{B. Inner product and projection operator}

There are several ways to derive the GLE, slightly different from each other. In this paper we follow that of Ref. 5. Let $A$ and $B$ be functions on $\mathcal{N}$. We define an inner product of the two functions by using the distribution

$$
(A \mid B)_{t} \stackrel{\text { def }}{=} \int A^{*}(\boldsymbol{q}, \boldsymbol{p}) B(\boldsymbol{q}, \boldsymbol{p}) \rho(\boldsymbol{q}, \boldsymbol{p}, t) \mathrm{d} \boldsymbol{q} \mathrm{d} \boldsymbol{p} .
$$

Note that the inner product is time dependent due to the time dependence of the distribution, in contrast to the usual treatments. ${ }^{3-5}$

We also define an inner product on the extended phase space. Let $\Phi$ and $\Psi$ be functions of $(\boldsymbol{q}, \boldsymbol{p}, t)$, and

$$
((\Phi \mid \Psi)) \stackrel{\text { def }}{=} \int \Phi^{*}(\boldsymbol{q}, \boldsymbol{p}, t) \Psi(\boldsymbol{q}, \boldsymbol{p}, t) \rho(\boldsymbol{q}, \boldsymbol{p}, t) \mathrm{d} \boldsymbol{q} \mathrm{d} \boldsymbol{p} \mathrm{d} t .
$$

The relation between these two kinds of inner product is easily seen:

$$
((\Phi \mid \Psi))=\int\left(\left.\Phi\right|_{t}|\Psi|_{t}\right)_{t} \mathrm{~d} t
$$

where the symbol $\left.\right|_{t}$ denotes the restriction of the function at $t$, that is, the function of $(\boldsymbol{q}, \boldsymbol{p}, t)$ is regarded as a function of $(\boldsymbol{q}, \boldsymbol{p})$ by fixing the value of $t$. Note that, in order for the integration over $t$ to converge, at least one of the functions $\Phi(\boldsymbol{q}, \boldsymbol{p}, t)$ and $\Psi(\boldsymbol{q}, \boldsymbol{p}, t)$ must decay sufficiently fast as $|t| \rightarrow \infty$. We must therefore restrict the range of functions for which the inner product is well-defined.
Note that the extended Liouvillian operator $\mathrm{i} \hat{\Lambda}$ is antiHermitian with respect to the inner product $((\cdot \mid \cdot))$ :

$$
(\mathrm{i} \hat{\Lambda})^{\dagger}=-\mathrm{i} \hat{\Lambda},
$$

where the symbol $\nmid$ denotes Hermitian conjugate. For any operator $\hat{\mathscr{F}}$ and functions $A$ and $B$, the Hermitian conjugate $\hat{\mathscr{F}}^{\dagger}$ is defined by

$$
((\hat{\mathscr{F}} A \mid B))=\left(\left(A \mid \hat{\mathscr{F}}^{\dagger} B\right)\right) .
$$

Equation (32) can be proved by partial integration and Eq. (28). In contrast, the operator i $\hat{\mathscr{L}}$ is not anti-Hermitian with respect to the inner product $(\cdot \mid \cdot)_{t}$ due to the nonstationarity of the distribution.

Let us next prepare a certain set of functions $f_{1}, f_{2}, \ldots, f_{m}$ on $\mathcal{N}$. These are functions of $(\boldsymbol{q}, \boldsymbol{p})$ only. The choice of this function set depends on what we want to project the system onto. We postpone the concrete choice until Sec. III C. Through the Gram-Schmidt orthonormalization with respect to the inner product $(\cdot \mid \cdot)_{t}$, we obtain an orthonormal set $\left\{\phi_{j, t}\right\}$ from $\left\{f_{j}\right\}$ :

$$
\begin{aligned}
\phi_{1, t} & =\frac{1}{\sqrt{\left(f_{1} \mid f_{1}\right)_{t}}} f_{1}, \\
\phi_{2, t} & =\frac{\tilde{\phi}_{2, t}}{\sqrt{\left(\tilde{\phi}_{2, t} \mid \tilde{\phi}_{2, t}\right)_{t}}}, \tilde{\phi}_{2, t}=f_{2}-\left(\phi_{1, t} \mid f_{2}\right)_{t} \phi_{1, t}
\end{aligned}
$$

so that

$$
\left(\phi_{i, t} \mid \phi_{j, t}\right)_{t}=\delta_{i j},
$$

where $\delta$ denotes Kronecker's delta. Note that $\phi_{j, t}$ depends on $t$ although the original function set $\left\{f_{j}(\boldsymbol{q}, \boldsymbol{p})\right\}$ does not depend on $t$. This is because of the $t$-dependence of the inner product.

We also prepare a complete orthonormal set along the $t$ axis $\left\{\chi_{n}\right\}$ :

$$
\begin{aligned}
\int \chi_{n}^{*}(t) \chi_{m}(t) \mathrm{d} t & =\delta_{m n}, \\
\sum_{n} \chi_{n}(t) \chi_{n}^{*}\left(t^{\prime}\right) & =\delta\left(t-t^{\prime}\right),
\end{aligned}
$$

where $\delta\left(t-t^{\prime}\right)$ is Dirac's delta function. An example of such a complete orthonormal set is Hermite functions, ${ }^{38}$ but we do not need to specify it concretely. We then define a function set $\left\{\Phi_{j, n}\right\}$ on the extended phase space by

$$
\Phi_{j, n}(\boldsymbol{q}, \boldsymbol{p}, t) \stackrel{\text { def }}{=} \phi_{j, t}(\boldsymbol{q}, \boldsymbol{p}) \chi_{n}(t) .
$$

They form an orthonormal set in the extended phase space with respect to the inner product $((\cdot \cdot \cdot))$ :

$$
\begin{aligned}
& \left(\left(\Phi_{i, m} \mid \Phi_{j, n}\right)\right) \\
& \quad=\int \phi_{i, t}^{*}(\boldsymbol{q}, \boldsymbol{p}) \chi_{m}^{*}(t) \phi_{j, t}(\boldsymbol{q}, \boldsymbol{p}) \chi_{n}(t) \rho(\boldsymbol{q}, \boldsymbol{p}, t) \mathrm{d} \boldsymbol{q} \mathrm{d} \boldsymbol{p} \mathrm{d} t \\
& \quad=\int \mathrm{d} t\left(\phi_{i, t} \mid \phi_{j, t}\right)_{t} \chi_{m}^{*}(t) \chi_{n}(t), \\
& \quad=\delta_{i j} \int \mathrm{d} t \chi_{m}^{*}(t) \chi_{n}(t), \\
& \quad=\delta_{i j} \delta_{m n} .
\end{aligned}
$$


The function set $\left\{\Phi_{j, n}\right\}$ is complete only for $t$, that is,

$$
\begin{aligned}
\sum_{j, n} & \Phi_{j, n}(\boldsymbol{q}, \boldsymbol{p}, t) \Phi_{j, n}^{*}\left(\boldsymbol{q}^{\prime}, \boldsymbol{p}^{\prime}, t^{\prime}\right) \\
& =\delta\left(t-t^{\prime}\right) \sum_{j} \phi_{j, t}(\boldsymbol{q}, \boldsymbol{p}) \phi_{j, t}^{*}\left(\boldsymbol{q}^{\prime}, \boldsymbol{p}^{\prime}\right) .
\end{aligned}
$$

By using the orthonormal sets we define a projection operator $\hat{\mathscr{P}}_{t}$ at $t$

$$
\hat{\mathscr{P}}_{t} F \stackrel{\text { def }}{=} \sum_{j} \phi_{j, t}\left(\phi_{j, t} \mid F\right)_{t}
$$

and a projection operator in the extended phase space

$$
\hat{\mathscr{P}} \Psi \stackrel{\text { def }}{=} \sum_{j, n} \Phi_{j, n}\left(\left(\Phi_{j, n} \mid \Psi\right)\right),
$$

for function $F=F(\boldsymbol{q}, \boldsymbol{p})$ and $\Psi=\Psi(\boldsymbol{q}, \boldsymbol{p}, t)$. From the completeness of $\left\{\chi_{n}\right\}$, it can be easily proved that

$$
\left.(\hat{\mathscr{P}} \Psi)\right|_{t}=\hat{\mathscr{P}}_{t}\left(\left.\Psi\right|_{t}\right),
$$

where the symbol $\left.\right|_{t}$ denotes the restriction of the function at $t$.

From the orthonormality of $\left\{\Phi_{j, n}\right\}$, it can be proved that

$$
\begin{aligned}
& \hat{\mathscr{P}}^{2}=\hat{\mathscr{P}}, \\
& \hat{\mathscr{P}}^{\dagger}=\hat{\mathscr{P}},
\end{aligned}
$$

where the dagger denotes the Hermitian conjugate, that is,

$$
((\hat{\mathscr{P}} \Psi \mid \Xi))=((\Psi \mid \hat{\mathscr{P}} \Xi)),
$$

for any functions $\Psi$ and $\Xi$.

\section{Irreversible generalized Langevin equation}

A next step for the derivation of GLE is to note the following operator identity: ${ }^{5}$

$$
\begin{aligned}
& \frac{\mathrm{d}}{\mathrm{d} \tau} \exp (\mathrm{i} \hat{\Lambda} \tau) \\
& =\exp (\mathrm{i} \hat{\Lambda} \tau) \hat{\mathscr{P}} \mathrm{i} \hat{\Lambda} \\
& \quad+\int_{0}^{\tau} \mathrm{d} s \exp (\mathrm{i} \hat{\Lambda}(\tau-s)) \hat{\mathscr{P}} \mathrm{i} \hat{\Lambda} \exp ((1-\hat{\mathscr{P}}) \mathrm{i} \hat{\Lambda} s) \\
& \quad \times(1-\hat{\mathscr{P}}) \mathrm{i} \hat{\Lambda}+\exp ((1-\hat{\mathscr{P}}) \mathrm{i} \hat{\Lambda} \tau)(1-\hat{\mathscr{P}}) \mathrm{i} \hat{\Lambda}
\end{aligned}
$$

This identity is obtained if we note that the integrand in the second term can be written as

$$
-\frac{\mathrm{d}}{\mathrm{d} s} \exp (\mathrm{i} \hat{\Lambda}(\tau-s)) \exp ((1-\hat{\mathscr{P}}) \mathrm{i} \hat{\Lambda} s)(1-\hat{\mathscr{P}}) \mathrm{i} \hat{\Lambda} \text {. }
$$

We take a certain set of physical quantities $\boldsymbol{A}$ $=\left(A_{1}, A_{2}, \ldots, A_{n}\right)$, which are functions of $(\boldsymbol{q}, \boldsymbol{p})$, and a complete set $\left\{f_{j}(\boldsymbol{A})\right\}$ of the functions of $\boldsymbol{A}$. The set is complete only in the function space of $\boldsymbol{A}$, but not necessarily in $(\boldsymbol{q}, \boldsymbol{p})$. By Gram-Schmidt normalization [Eq. (34)] we construct an orthonormal set $\left\{\phi_{j, t}(\boldsymbol{A})\right\}$ which is complete in the function space of $\boldsymbol{A}$. From the orthonormality and the completeness, it is proved (in Appendix A) that

$$
P_{t}(\boldsymbol{a}) \sum_{j} \phi_{j, t}^{*}\left(\boldsymbol{a}^{\prime}\right) \phi_{j, t}(\boldsymbol{a})=\delta\left(\boldsymbol{a}-\boldsymbol{a}^{\prime}\right),
$$

where $P_{t}(\boldsymbol{a})$ is the distribution of the quantity $\boldsymbol{A}$ at $t$ :

$$
P_{t}(\boldsymbol{a}) \stackrel{\text { def }}{=} \int \delta(\boldsymbol{A}(\boldsymbol{q}, \boldsymbol{p})-\boldsymbol{a}) \rho(\boldsymbol{q}, \boldsymbol{p}, t) \mathrm{d} \boldsymbol{q} \mathrm{d} \boldsymbol{p} .
$$

Here we use the upper case $\boldsymbol{A}=\boldsymbol{A}(\boldsymbol{q}, \boldsymbol{p})$ to denote the phase space function, and the lower case $\boldsymbol{a}$ for its numerical values.

When we let Eq. (45) operate on $\boldsymbol{A}$, the left hand side is

$$
\frac{\mathrm{d}}{\mathrm{d} \tau} \boldsymbol{A}(\tau)
$$

where $\boldsymbol{A}(\tau)$ is the time evolution of $\boldsymbol{A}$ [Eq. (26)]. Note this derivative by $\tau$ is a differentiation by the first argument $\tau$ of $\boldsymbol{A}(\tau ; \boldsymbol{q}, \boldsymbol{p})$ with the initial condition $(\boldsymbol{q}, \boldsymbol{p})$ kept intact. The first term on the right hand side becomes

$$
\begin{aligned}
& \exp (\mathrm{i} \hat{\Lambda} \tau) \hat{\mathscr{P}}_{\mathrm{i}} \hat{\Lambda} \boldsymbol{A} \\
&=\exp (\mathrm{i} \hat{\Lambda} \tau) \hat{\mathscr{P}}_{\dot{\boldsymbol{A}}}, \\
&=\exp (\mathrm{i} \hat{\Lambda} \tau) \sum_{j} \phi_{j, t}(\boldsymbol{A})\left(\phi_{j, t} \mid \dot{\boldsymbol{A}}\right)_{t}, \\
&=\sum_{j} \phi_{j, t+\tau}(\boldsymbol{A}(\tau))\left(\phi_{j, t+\tau} \mid \dot{\boldsymbol{A}}\right)_{t+\tau}, \\
&=\sum_{j} \phi_{j, t+\tau}(\boldsymbol{A}(\tau)) \\
& \quad \times \int_{\mathrm{d} \boldsymbol{q}^{\prime} \mathrm{d} \boldsymbol{p}^{\prime} \rho\left(\boldsymbol{q}^{\prime}, \boldsymbol{p}^{\prime}, t+\tau\right) \phi_{j, t+\tau}\left(\boldsymbol{A}\left(\boldsymbol{q}^{\prime}, \boldsymbol{p}^{\prime}\right)\right) \dot{\boldsymbol{A}}\left(\boldsymbol{q}^{\prime}, \boldsymbol{p}^{\prime}\right),} P_{t+\tau}(\boldsymbol{A}(\tau))^{-1} \\
& \times \int \mathrm{d} \boldsymbol{q}^{\prime} \mathrm{d} \boldsymbol{p}^{\prime} \rho\left(\boldsymbol{q}^{\prime}, \boldsymbol{p}^{\prime}, t+\tau\right) \delta\left(\boldsymbol{A}\left(\boldsymbol{q}^{\prime}, \boldsymbol{p}^{\prime}\right)-\boldsymbol{A}(\tau)\right) \\
& \times \dot{\boldsymbol{A}}\left(\boldsymbol{q}^{\prime}, \boldsymbol{p}^{\prime}\right), \\
&=\langle\dot{\boldsymbol{A}} ; \boldsymbol{A}(\tau)\rangle_{t+\tau},
\end{aligned}
$$

where we have used Eq. (42) at the second equality, and

$$
\dot{A} \stackrel{\text { def. }}{=} \hat{\Lambda} \boldsymbol{A}
$$

is the time derivative of $\boldsymbol{A}$. At the third equality, noting that the operator $\exp (\mathrm{i} \hat{\Lambda} \tau)$ gives the time evolution in the extended phase space, we changed the arguments $t$ of the functions accordingly. At the fifth equality Eq. (47) was used. Finally we utilize the symbol $\langle F ; \boldsymbol{a}\rangle_{t}$ that stands for the average of $F$ with a fixed value of $\boldsymbol{A}=\boldsymbol{a}$ over the distribution at $t$ :

$$
\langle F ; \boldsymbol{a}\rangle_{t} \stackrel{\text { def }}{=} P_{t}(\boldsymbol{a})^{-1} \int \mathrm{d} \boldsymbol{q} \mathrm{d} \boldsymbol{p} \rho(\boldsymbol{q}, \boldsymbol{p}, t) \delta(\boldsymbol{A}-\boldsymbol{a}) F(\boldsymbol{q}, \boldsymbol{p}),
$$

and $\langle\dot{\boldsymbol{A}} ; \boldsymbol{A}(\tau)\rangle_{t+\tau}$ is a substitution of $\boldsymbol{A}(\tau)$ in the place of $\boldsymbol{a}$.

As for the third term on the right hand side of Eq. (45) operating on $\boldsymbol{A}$, we express it by a function $\boldsymbol{\xi}(\tau)$ :

$$
\boldsymbol{\xi}(\tau)=\boldsymbol{\xi}(\tau ; \boldsymbol{q}, \boldsymbol{p}, t) \stackrel{\text { def }}{=} \exp ((1-\hat{\mathscr{P}}) \mathrm{i} \hat{\Lambda} \tau)(1-\hat{\mathscr{P}}) \dot{\boldsymbol{A}} .
$$


The $\tau$-dependence of the function $\boldsymbol{\xi}(\tau)$ is given by the evolution under $(1-\hat{\mathscr{P}})$ i $\hat{\Lambda}$, in contrast to Eq. (26). Note, because of $\hat{\mathscr{P}}^{2}=\hat{\mathscr{P}}$,

$$
\boldsymbol{\xi}(\tau)=(1-\hat{\mathscr{P}}) \boldsymbol{\xi}(\tau)
$$

which will be used in what follows. Then the integrand of the second term on the right hand side of Eq. (45) becomes

$$
\begin{aligned}
& \exp (\mathrm{i} \hat{\Lambda}(\tau-s)) \hat{\mathscr{P}} \mathrm{i} \hat{\Lambda} \xi(s), \\
& \quad=\exp (\mathrm{i} \hat{\Lambda}(\tau-s)) \sum_{j, n} \Phi_{j, n}\left(\left(\Phi_{j, n} \mid \mathrm{i} \hat{\Lambda} \xi(s)\right)\right), \\
& \quad=\exp (\mathrm{i} \hat{\Lambda}(\tau-s)) \sum_{j, n} \phi_{j, t}(\boldsymbol{A}) \chi_{n}(t)\left(\left(\Phi_{j, n} \mid \mathrm{i} \hat{\Lambda} \xi(s)\right)\right), \\
& =\sum_{j, n} \phi_{j, t+\tau-s}(A(\tau-s)) \chi_{n}(t+\tau-s)\left(\left(\Phi_{j, n} \mid \mathrm{i} \hat{\Lambda} \xi(s)\right)\right) .
\end{aligned}
$$

Since $i \hat{\Lambda}$ is anti-Hermitian, we have

$$
\left(\left(\Phi_{j, n} \mid \mathrm{i} \hat{\Lambda} \boldsymbol{\xi}(s)\right)\right)=-\left(\left(\mathrm{i} \hat{\Lambda} \Phi_{j, n} \mid \boldsymbol{\xi}(s)\right)\right) .
$$

Further,

$$
\begin{aligned}
& {[\exp ((1-\hat{\mathscr{P}}) \mathrm{i} \hat{\Lambda} \tau)(1-\hat{\mathscr{P}})]^{\dagger}} \\
& \quad=[\sum_{n=0}^{\infty} \frac{\tau^{n}}{n !} \underbrace{(1-\hat{\mathscr{P}}) \mathrm{i} \hat{\Lambda} \cdots(1-\hat{\mathscr{P}}) \mathrm{i} \hat{\Lambda}}_{n}(1-\hat{\mathscr{P}})]^{\dagger}, \\
& \quad=\sum_{n=0}^{\infty} \frac{\tau^{n}}{n !}(1-\hat{\mathscr{P}}) \underbrace{(-\mathrm{i} \hat{\Lambda})(1-\hat{\mathscr{P}}) \cdots(-\mathrm{i} \hat{\Lambda})(1-\hat{\mathscr{P}})}_{n}, \\
& \quad=\sum_{n=0}^{\infty} \frac{(-\tau)^{n}}{n !} \underbrace{(1-\hat{\mathscr{P}}) \mathrm{i} \hat{\Lambda}(1-\hat{\mathscr{P}}) \cdots \mathrm{i} \hat{\Lambda}}_{n}(1-\hat{\mathscr{P}}), \\
& \quad=\exp (-(1-\hat{\mathscr{P}}) \mathrm{i} \hat{\Lambda} \tau)(1-\hat{\mathscr{P}}) .
\end{aligned}
$$

We use this to the last part of Eq. (56) and note Eqs. (53) and (54) to obtain

$$
\begin{aligned}
& -\left(\left(\mathrm{i} \hat{\Lambda} \Phi_{j, n} \mid \boldsymbol{\xi}(s)\right)\right), \\
& \quad=-\left(\left(\mathrm{i} \hat{\Lambda} \Phi_{j, n} \mid \exp ((1-\hat{\mathscr{P}}) \mathrm{i} \hat{\Lambda}(s-\tau)) \xi(\tau)\right)\right), \\
& =-\left(\left(\mathrm{i} \hat{\Lambda} \Phi_{j, n} \mid \exp ((1-\hat{\mathscr{P}}) \mathrm{i} \hat{\Lambda}(s-\tau))(1-\hat{\mathscr{P}}) \boldsymbol{\xi}(\tau)\right)\right), \\
& =-\left(\left(\exp ((1-\hat{\mathscr{P}}) \mathrm{i} \hat{\Lambda}(\tau-s))(1-\hat{\mathscr{P}}) \mathrm{i} \hat{\Lambda} \Phi_{j, n} \mid \boldsymbol{\xi}(\tau)\right)\right) .
\end{aligned}
$$

Since $\Phi_{j, n}=\phi_{j, t}(\boldsymbol{A}) \chi_{n}(t)$ is a function only of $\boldsymbol{A}$ and $t$, we have

$$
\begin{aligned}
(1-\hat{\mathscr{P}}) \mathrm{i} \hat{\Lambda} \Phi_{j, n} & =(1-\hat{\mathscr{P}})\left(\frac{\partial \Phi_{j, n}}{\partial \boldsymbol{A}} \cdot \dot{\boldsymbol{A}}+\frac{\partial \Phi_{j, n}}{\partial t}\right) \\
& =\frac{\partial \Phi_{j, n}}{\partial \boldsymbol{A}} \cdot \boldsymbol{\xi}(0),
\end{aligned}
$$

where we have used Eqs. (B1) [i.e., $\hat{\mathscr{P}}\left(\partial \Phi_{j, n} / \partial t\right)$ $=\partial \Phi_{j, n} / \partial t$ ], (B3) [i.e., $\left.\hat{\mathscr{P}}\left\{\left(\partial \Phi_{j, n} / \partial \boldsymbol{A}\right) \dot{\boldsymbol{A}}\right\}=\left(\partial \Phi_{j, n} / \partial \boldsymbol{A}\right) \hat{\mathscr{P}} \dot{\boldsymbol{A}}\right]$ in Appendix B, Eq. (53) [i.e., $(1-\hat{\mathscr{P}}) \dot{\boldsymbol{A}}=\boldsymbol{\xi}(0)$ ], and the dot denotes the inner product of vectors.
Further, by using Eq. (B5),

$$
\begin{aligned}
& \exp ((1-\hat{\mathscr{P}}) \mathrm{i} \hat{\Lambda}(\tau-s)) \frac{\partial \Phi_{j, n}}{\partial \boldsymbol{A}} \cdot \boldsymbol{\xi}(0) \\
= & \chi_{n}(t+\tau-s) \tilde{\xi}_{j}(\tau-s),
\end{aligned}
$$

with

$$
\tilde{\xi}_{j}(\sigma) \stackrel{\text { def }}{=} \exp ((1-\hat{\mathscr{P}}) \mathrm{i} \hat{\Lambda} \sigma) \frac{\partial \phi_{j, t}}{\partial \boldsymbol{A}} \cdot \boldsymbol{\xi}(0) .
$$

Equation (55) thus becomes

$$
\begin{aligned}
\sum_{j, n} \phi_{j, t+\tau-s}(\boldsymbol{A}(\tau-s)) \chi_{n}(t+\tau-s)\left(\left(\Phi_{j, n} \mid \mathrm{i} \hat{\Lambda} \boldsymbol{\xi}(s)\right)\right) \\
=\sum_{j, n} \phi_{j, t+\tau-s}(\boldsymbol{A}(\tau-s)) \chi_{n}(t+\tau-s) \\
\quad \times\left(\left(\chi_{n}(t+\tau-s) \tilde{\xi}_{j}(\tau-s) \mid \boldsymbol{\xi}(\tau)\right)\right) \\
=-\sum_{j, n} \phi_{j, t+\tau-s}(\boldsymbol{A}(\tau-s)) \chi_{n}(t+\tau-s) \\
\quad \times \int_{\mathrm{d} \boldsymbol{q}^{\prime} \mathrm{d} \boldsymbol{p}^{\prime} \mathrm{d} t^{\prime} \rho\left(\boldsymbol{q}^{\prime}, \boldsymbol{p}^{\prime}, t^{\prime}\right) \chi_{n}\left(t^{\prime}+\tau-s\right)} \\
\quad \times \tilde{\xi}_{j}\left(\tau-s ; \boldsymbol{q}^{\prime}, \boldsymbol{p}^{\prime}, t^{\prime}\right) \boldsymbol{\xi}\left(\tau ; \boldsymbol{q}^{\prime}, \boldsymbol{p}^{\prime}, t^{\prime}\right) \\
=-\sum_{j} \phi_{j, t+\tau-s}(\boldsymbol{A}(\tau-s)) \\
\quad \times \int_{\mathrm{d}} \mathrm{d} \boldsymbol{q}^{\prime} \mathrm{d} \boldsymbol{p}^{\prime} \rho\left(\boldsymbol{q}^{\prime}, \boldsymbol{p}^{\prime}, t\right) \tilde{\xi}_{j}\left(\tau-s ; \boldsymbol{q}^{\prime}, \boldsymbol{p}^{\prime}, t\right) \boldsymbol{\xi}\left(\tau ; \boldsymbol{q}^{\prime}, \boldsymbol{p}^{\prime}, t\right) \\
=-\sum_{j} \phi_{j, t+\tau-s}(\boldsymbol{A}(\tau-s))\left\langle\tilde{\xi}_{j}(\tau-s) \xi(\tau)\right\rangle_{t},
\end{aligned}
$$

where $\langle\cdot\rangle_{t}$ denotes the average over the distribution $\rho(\boldsymbol{q}, \boldsymbol{p}, t)$ at $t$.

Remember that

$$
\boldsymbol{A}(\tau)=\boldsymbol{A}(\tau ; \boldsymbol{q}, \boldsymbol{p}, t)=\exp (\mathrm{i} \hat{\Lambda} \tau) \boldsymbol{A}
$$

gives the value of $\boldsymbol{A}$ at $\tau$, as a function of the initial condition $(\boldsymbol{q}, \boldsymbol{p}, t)$. Similarly, $\boldsymbol{\xi}(\tau)=\boldsymbol{\xi}(\tau ; \boldsymbol{q}, \boldsymbol{p}, t)$ gives the value of $\boldsymbol{\xi}$ at $\tau$, as a function of the initial condition $(\boldsymbol{q}, \boldsymbol{p}, t)$. Since the initial condition of $t$ is taken as $\left.t\right|_{\tau=0}=0$, we only need the values at $t=0$. Thus we finally obtain the iGLE by substituting $t=0$ in Eqs. (50) and (62):

$$
\begin{aligned}
\frac{\mathrm{d}}{\mathrm{d} \tau} \boldsymbol{A}(\tau)= & \langle\dot{\boldsymbol{A}} ; \boldsymbol{A}(\tau)\rangle_{\tau}+\boldsymbol{\xi}(\tau) \\
& -\int_{0}^{\tau} \mathrm{d} s \sum_{j} \phi_{j, \tau-s}(\boldsymbol{A}(\tau-s))\left\langle\tilde{\xi}_{j}(\tau-s) \boldsymbol{\xi}(\tau)\right\rangle_{0} .
\end{aligned}
$$

A useful identity about the function $\xi$ is that it has no correlation with the chosen physical quantity $\boldsymbol{A}$ :

$$
\langle f(\boldsymbol{A}) \boldsymbol{\xi}(s)\rangle_{0}=0,
$$


for any function $f$ of only $\boldsymbol{A}$. The proof is as follows. First, we have

$$
\begin{aligned}
\sum_{n} & \chi_{n}(0)\left(\left(\chi_{n} f(\boldsymbol{A}) \mid \boldsymbol{\xi}(s)\right)\right), \\
= & \sum_{n} \chi_{n}(0) \int \mathrm{d} \boldsymbol{q}^{\prime} \mathrm{d} \boldsymbol{p}^{\prime} \mathrm{d} t^{\prime} \chi_{n}\left(t^{\prime}\right) f\left(\boldsymbol{A}\left(\boldsymbol{q}^{\prime}, \boldsymbol{p}^{\prime}\right)\right) \xi(s) \\
& \times \rho\left(\boldsymbol{q}^{\prime}, \boldsymbol{p}^{\prime}, t^{\prime}\right), \\
= & \int \mathrm{d} \boldsymbol{q}^{\prime} \mathrm{d} \boldsymbol{p}^{\prime} f\left(\boldsymbol{A}\left(\boldsymbol{q}^{\prime}, \boldsymbol{p}^{\prime}\right)\right) \xi(s) \rho\left(\boldsymbol{q}^{\prime}, \boldsymbol{p}^{\prime}, 0\right), \\
= & \langle f(\boldsymbol{A}) \boldsymbol{\xi}(s)\rangle_{0} .
\end{aligned}
$$

On the other hand, because of Eqs. (54) and (B1),

$$
\begin{aligned}
\sum_{n} \chi_{n}(0)\left(\left(\chi_{n} f(\boldsymbol{A}) \mid \boldsymbol{\xi}(s)\right)\right), \\
=\sum_{n} \chi_{n}(0)\left(\left(\chi_{n} f(\boldsymbol{A}) \mid(1-\hat{\mathscr{P}}) \boldsymbol{\xi}(s)\right)\right), \\
=\sum_{n} \chi_{n}(0)\left(\left((1-\hat{\mathscr{P}}) \chi_{n} f(\boldsymbol{A}) \mid \boldsymbol{\xi}(s)\right)\right), \\
=0 .
\end{aligned}
$$

We also note that, in stationary states, $\left\langle\tilde{\xi}_{j}(\tau-s) \xi(\tau)\right\rangle_{0}$ in the integrand of Eq. (64) depends only on the time difference:

$$
\left\langle\tilde{\xi}_{j}(\tau-s) \boldsymbol{\xi}(\tau)\right\rangle_{0} \stackrel{\text { stationary }}{=}\left\langle\tilde{\xi}_{j}(0) \boldsymbol{\xi}(s)\right\rangle_{0} .
$$

The proof is given in Appendix C.

\section{Second-order equation of motion}

As a special case, we here take physical quantities

$$
\begin{aligned}
& Q=Q(\boldsymbol{q}, \boldsymbol{p}), \\
& V=V(\boldsymbol{q}, \boldsymbol{p}) \stackrel{\text { def. }}{=} \mathrm{i} \hat{\Lambda} Q=\mathrm{i} \hat{\mathscr{L}} Q,
\end{aligned}
$$

and set $\boldsymbol{A}=(Q, V)$. Let the first function of the complete set $\left\{f_{j}(Q, V)\right\}$ be

$$
f_{1}(Q, V)=V,
$$

and approximate the iGLE [Eq. (64)] by truncating the sum at the first term. The first step of the Gram-Schmidt orthonormalization [Eq. (34)] goes

$$
\phi_{1, t}=\frac{1}{\left\langle V^{2}\right\rangle_{t}^{1 / 2}} V .
$$

Substituting this into Eq. (64) yields

$$
\begin{aligned}
\frac{\mathrm{d}}{\mathrm{d} \tau} Q(\tau)= & \langle\dot{Q} ; Q(\tau), V(\tau)\rangle_{\tau}+\xi_{Q}(\tau) \\
& -\int_{0}^{\tau} \mathrm{d} s \frac{1}{\left\langle V^{2}\right\rangle_{\tau-s}^{1 / 2}} V(\tau-s)\left\langle\tilde{\xi}_{1}(\tau-s) \xi_{Q}(\tau)\right\rangle_{0}, \\
\frac{\mathrm{d}}{\mathrm{d} \tau} V(\tau)= & \langle\dot{V} ; Q(\tau), V(\tau)\rangle_{\tau}+\xi_{V}(\tau) \\
& -\int_{0}^{\tau} \mathrm{d} s \frac{1}{\left\langle V^{2}\right\rangle_{\tau-s}^{1 / 2}} V(\tau-s)\left\langle\tilde{\xi}_{1}(\tau-s) \xi_{V}(\tau)\right\rangle_{0},
\end{aligned}
$$

where we have introduced the notation $\xi=\left(\xi_{Q}, \xi_{V}\right)$. However, in this case we have a particular choice of the physical quantities such that $\dot{Q}=V$, and $\langle\dot{Q} ; Q(\tau), V(\tau)\rangle_{\tau}$ is an average of $\dot{Q}$ with fixed values of $Q(\tau)$ and $V(\tau)$, which is simply $V(\tau)$. Also, from the definition of $\boldsymbol{\xi}$,

$$
\xi_{Q}(\tau)=\exp ((1-\hat{\mathscr{P}}) \mathrm{i} \hat{\Lambda} \tau)(1-\hat{\mathscr{P}}) V=0,
$$

since $(1-\hat{\mathscr{P}}) V=0$ because of Eq. (B1). The first equation of Eq. (72) therefore reduces to

$$
\frac{\mathrm{d}}{\mathrm{d} \tau} Q(\tau)=V(\tau)
$$

Next, from the definition of $\tilde{\xi}$ [Eq. (61)], we have

$$
\begin{aligned}
\tilde{\xi}_{1}(\tau) & =\exp ((1-\hat{\mathscr{P}}) \mathrm{i} \hat{\Lambda} \tau)\left[\frac{\partial \phi_{1, t}}{\partial Q} \xi_{Q}(0)+\frac{\partial \phi_{1, t}}{\partial V} \xi_{V}(0)\right] \\
& =\exp ((1-\hat{\mathscr{P}}) \mathrm{i} \hat{\Lambda} \tau) \frac{1}{\left\langle V^{2}\right\rangle_{t}^{1 / 2}} \xi_{V}(0) \\
& =\frac{1}{\left\langle V^{2}\right\rangle_{t+\tau}^{1 / 2}} \exp ((1-\hat{\mathscr{P}}) \mathrm{i} \hat{\Lambda} \tau) \xi_{V}(0) \\
& =\frac{1}{\left\langle V^{2}\right\rangle_{t+\tau}^{1 / 2}} \xi_{V}(\tau)
\end{aligned}
$$

[see Eqs. (53) and (B5)]. By substituting $t=0$, Eq. (72) becomes

$$
\begin{aligned}
\frac{\mathrm{d}}{\mathrm{d} \tau} V(\tau)= & \langle\dot{V} ; Q(\tau), V(\tau)\rangle_{\tau}+\xi_{V}(\tau) \\
& -\int_{0}^{\tau} \mathrm{d} s \frac{1}{\left\langle V^{2}\right\rangle_{\tau-s}} V(\tau-s)\left\langle\xi_{V}(\tau-s) \xi_{V}(\tau)\right\rangle_{0} .
\end{aligned}
$$

Combining this with Eq. (74) we finally obtain

$$
\begin{aligned}
\frac{\mathrm{d}^{2}}{\mathrm{~d} \tau^{2}} Q(\tau)= & \langle\ddot{Q} ; Q(\tau), \dot{Q}(\tau)\rangle_{\tau} \\
& -\int_{0}^{\tau} \mathrm{d} \tau^{\prime} \gamma\left(\tau, \tau^{\prime}\right) \dot{Q}\left(\tau^{\prime}\right)+\xi_{V}(\tau),
\end{aligned}
$$

with the generalized friction kernel defined by

$$
\gamma\left(\tau, \tau^{\prime}\right) \stackrel{\text { def }}{=} \frac{\left\langle\xi_{V}(\tau) \xi_{V}\left(\tau^{\prime}\right)\right\rangle_{0}}{\left\langle\dot{Q}^{2}\right\rangle_{\tau^{\prime}}},
$$

corresponding to what Refs. 28,29, and 34 called the generalized fluctuation-dissipation theorem.

When the physical quantity $Q$ can be interpreted as position, and $V$ velocity, the first term on the right hand side of Eq. (77) is called the mean force, because it gives the value of the acceleration averaged over the distribution of the surroundings (degrees of freedom other than $Q$ ). Similarly, the second term is the "frictional" force representing the response of the surroundings to the motion of the system described by $Q$ and $\dot{Q}$. The third term $\xi_{V}(\tau)$ is called random force. It represents the deviation from the average of the force exerted by the surrounding and felt by the system. Although the value of $\xi_{V}(\tau)$ is given by the deterministic equation of motion [Eq. (22)] for the total system, the randomness comes from the fact that we know the initial condition of the surroundings only as the distribution $\rho(\boldsymbol{q}, \boldsymbol{p}, 0)$. The value of $\xi_{V}(\tau)$ has 
uncertainty inherent to the limited information regarding the environment, that is, all what we can know is just the distribution of $(\boldsymbol{q}, \boldsymbol{p})$ at time $t=0$. The statistical property of $\xi_{V}(\tau)$ is given, for example, by the generalized fluctuationdissipation theorem Eq. (78). Moreover, as special cases of Eq. (65), we can prove that the average of the random force and its correlation with the initial condition of $(Q, \dot{Q})$ vanish:

$$
\begin{aligned}
\left\langle\xi_{V}(\tau)\right\rangle_{0} & =0, \\
\left\langle\xi_{V}(\tau) Q\right\rangle_{0} & =0, \\
\left\langle\xi_{V}(\tau) \dot{Q}\right\rangle_{0} & =0,
\end{aligned}
$$

and so forth.

Note that, in the equilibrium, the average of the square of the velocity is the temperature

$$
\left\langle\dot{Q}^{2}\right\rangle=k_{\mathrm{B}} T
$$

and $\gamma\left(\tau, \tau^{\prime}\right)$ depends only on $\left(\tau-\tau^{\prime}\right)$ because of Eq. (68). In the same situation, the mean force also becomes the equilibrium average of $\ddot{Q}$. Therefore Eq. (77) reduces to the usual one. In Eq. (78) for nonstationary cases, we have the average of the square of the velocity at time $\tau^{\prime}$, which may be interpreted as an effective temperature at time $\tau^{\prime}$ in the nonstationary process. Note also that the form of the friction term in Eq. (77) is the same with Eq. (7) which was obtained by the heuristic consideration. ${ }^{28-34}$ The generalized fluctuation-dissipation theorem proposed ${ }^{28-34}$ has also been proved on general footing here. Note also that, compared to the equilibrium case, we need to reinterpret the "temperature" of the nonstationary process as the average of the square of the velocity. Moreover, in contrast to the previous treatments, ${ }^{28-34}$ the mean force $\langle\ddot{Q} ; Q(\tau), \dot{Q}(\tau)\rangle_{\tau}$ depends generally on both the position $Q(\tau)$ and the velocity $\dot{Q}(\tau)$, and its functional form is also time dependent due to \langle\rangle$_{\tau}$.

The differentiation of "time $\tau$ " from the "canonical coordinate $t$ " in the extended phase space was utilized in the above derivation based on the projection operator formalism. However, once we have obtained the equation of motion in the form of Eq. (64) or (77), we can change the argument from $\tau$ to $t$ to obtain the usual expression reviewed in Sec. II, since their values are in fact the same by Eq. (24).

\section{SIMPLE EXAMPLE}

In this section we illustrate the concept of iGLE derived in Sec. III by using a simple numerical example. The example consists of a harmonic oscillator $\left(q_{1}, p_{1}\right)$ that is regarded as the "system," and another harmonic oscillator $\left(q_{2}, p_{2}\right)$ coupled to the system and an outer bath of Langevin type:

$$
\begin{aligned}
\frac{\mathrm{d}}{\mathrm{d} t} q_{1} & =p_{1}, \\
\frac{\mathrm{d}}{\mathrm{d} t} p_{1} & =-\omega_{1}^{2} q_{1}+a \omega_{2}^{2}\left(q_{2}-a q_{1}\right),
\end{aligned}
$$

$$
\begin{aligned}
\frac{\mathrm{d}}{\mathrm{d} t} q_{2} & =p_{2}, \\
\frac{\mathrm{d}}{\mathrm{d} t} p_{2} & =-\zeta p_{2}-\omega_{2}^{2}\left(q_{2}-a q_{1}\right)+\eta(t),
\end{aligned}
$$

where $\omega_{1}$ and $\omega_{2}$ are the frequencies of the two oscillators, and $a$ is the coupling constant between modes 1 and 2 . The coupling of $\left(q_{2}, p_{2}\right)$ to the outer bath is represented by the friction constant $\zeta$ and the Langevin-type random force $\eta(t)$. They are related by fluctuation-dissipation theorem:

$$
\left\langle\eta(t) \eta\left(t^{\prime}\right)\right\rangle=2 k_{\mathrm{B}} T_{\mathrm{o}} \zeta \delta\left(t-t^{\prime}\right),
$$

with the temperature $T_{\mathrm{o}}$ of the outer bath.

The "bath" in this model consists of the harmonic oscillator $\left(q_{2}, p_{2}\right)$, which is in direct contact to the system $\left(q_{1}, p_{1}\right)$, and the outer bath. While the outer bath is assumed to be in thermal equilibrium with the temperature $T_{\mathrm{o}}$, the initial condition of $\left(q_{2}, p_{2}\right)$ can be given by a nonequilibrium distribution. Here we give the initial condition of $\left(q_{2}, p_{2}\right)$ with a vibrational excitation with a temperature $T_{2}$ higher than the bulk temperature $T_{0}$. The dynamics of the system $\left(q_{1}, p_{1}\right)$ is subject to the effects of the nonequilibrium bath such as energy flow from the excited bath mode and time-dependent rate of energy dissipation to the outer bath. Its description must be based on the equation of motion for $\left(q_{1}, p_{1}\right)$ with the nonstationarity of the bath taken into account. Thus we reduce Eq. (81) to an equation only of $q_{1}$ by the procedure of Sec. III to obtain the following iGLE:

$$
\ddot{q}_{1}=f\left(q_{1}, \dot{q}_{1}, t\right)-\int_{0}^{t} \gamma\left(t, t^{\prime}\right) \dot{q}_{1}\left(t^{\prime}\right) \mathrm{d} t^{\prime}+\xi(t),
$$

where $f\left(q_{1}(t), \dot{q}_{1}(t), t\right)$ is the mean force at time $t$ (averaged over the given distribution of the bath), and $\gamma\left(t, t^{\prime}\right)$ is the timedependent friction kernel that is related to the random force $\xi(t)$ by [as Eq. (78)]

$$
\left\langle\xi(t) \xi\left(t^{\prime}\right)\right\rangle_{0}=\left\langle\dot{q}_{1}^{2}\right\rangle_{t^{\prime}} \gamma\left(t, t^{\prime}\right),
$$

where the bracket denotes the average over the given distribution of the bath. In this section we use the symbol $t$ for the independent parameter in place of $\tau$.

As the distribution $\rho$, we take a Boltzmann-type distribution of initial temperature $T_{1}^{\text {initial }}$ and $T_{2}^{\text {initial }}$ for the two modes:

$$
\begin{array}{r}
\rho\left(q_{1}, q_{2}, p_{1}, p_{2}, 0\right) \propto \exp \left[-\frac{1}{k_{\mathrm{B}} T_{1}^{\text {initial }}}\left(\frac{1}{2}{p_{1}}^{2}+\frac{\omega_{1}^{2}}{2} q_{1}^{2}\right)\right. \\
\left.-\frac{1}{k_{\mathrm{B}} T_{2}^{\text {initial }}}\left(\frac{1}{2} p_{2}{ }^{2}+\frac{\omega_{2}^{2}}{2} q_{2}^{2}\right)\right],
\end{array}
$$

and its time evolution $\rho\left(q_{1}, q_{2}, p_{1}, p_{2}, t\right)$ due to Eq. (81).

The numerical values of the parameters are taken as $\omega_{1}$ $=1, \omega_{2}=2, a=1 / 2, \zeta=1 / 5, k_{\mathrm{B}} T_{1}^{\text {initial }}=1 / 2, k_{\mathrm{B}} T_{2}^{\text {initial }}$ $=3 / 2$, and $k_{\mathrm{B}} T_{\mathrm{o}}=1 / 2$. Namely, the vibrational mode $\left(q_{2}, p_{2}\right)$ is initially excited compared to $\left(q_{1}, p_{1}\right)$ and the outer bath. It will then be de-excited and relax to the outer bath temperature $k_{\mathrm{B}} T_{\mathrm{o}}$. Details of calculation of the projection operator, the mean force, and the friction kernels are given in a supplementary material file. ${ }^{39}$ 


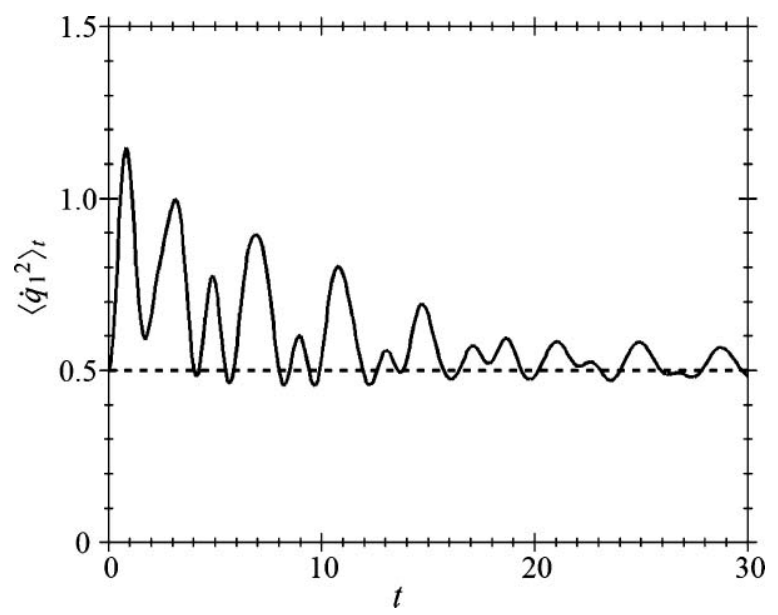

FIG. 1. Average of the squared velocity $\left\langle\dot{q}_{1}^{2}\right\rangle_{t}$ as a function of time.

Figure 1 shows the time evolution of the average of the squared velocity $\left\langle\dot{q}_{1}^{2}\right\rangle_{t}$, or the "effective temperature," which appears in the fluctuation-dissipation relation Eq. (84). At $t=0$, it is equal to $k_{\mathrm{B}} T_{1}^{\text {initial }}=1 / 2$, and then it suddenly increases due to the energy flow from the $\left(q_{2}, p_{2}\right)$ mode that was initially excited. As time goes further, the value $\left\langle\dot{q}_{1}^{2}\right\rangle_{t}$ decreases gradually with some oscillatory behavior, and finally relaxes to the temperature $k_{\mathrm{B}} T_{\mathrm{O}}=1 / 2$ of the outer bath.

Since the original equations of motion [Eq. (81)] are linear, it can be shown that the mean force in the iGLE (83) is also linear in $q_{1}(t)$ and $\dot{q}_{1}(t)$ :

$$
f\left(q_{1}, \dot{q}_{1}, t\right)=\alpha(t) q_{1}+\beta(t) \dot{q}_{1},
$$

with time-dependent coefficients $\alpha(t)$ and $\beta(t)$. The timeevolution of the coefficients obtained by numerical calculations are shown in Fig. 2.

In contrast to the equilibrium version, the mean force in the iGLE depends also on the velocity, and its functional form shows oscillatory behavior as a function of time (Fig. 2). As time goes to infinity, the coefficients converge to the equilibrium values: $\alpha(t) \rightarrow-\omega_{1}^{2}=-1$ and $\beta(t) \rightarrow 0$, where we

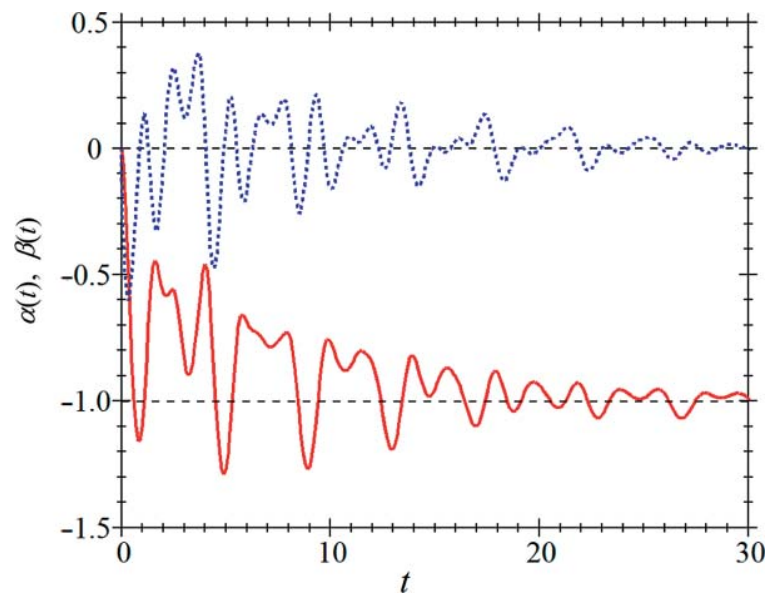

FIG. 2. Coefficients of the mean force as functions of time. The solid curve shows the coefficient $\alpha(t)$ of $q_{1}$, and the dotted curve shows the coefficient $\beta(t)$ of $\dot{q}_{1}$.
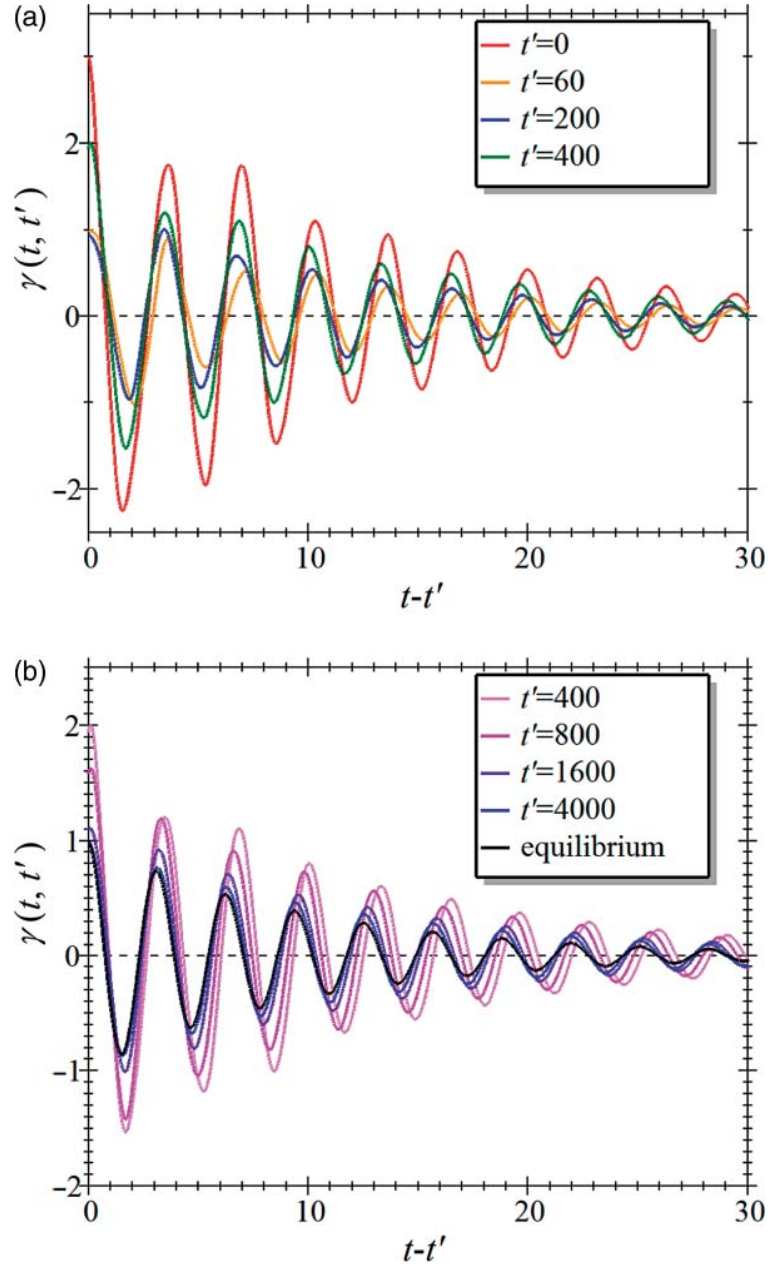

FIG. 3. Friction kernel as a function of time difference. Functional forms of the friction kernel are shown for various values of the initial time. The darkest curve in the lower panel shows the equilibrium friction kernel $\gamma_{\mathrm{eq}}$.

note that the equilibrium version of GLE can be obtained in the usual way ${ }^{35}$ and reads

$$
\ddot{q}_{1}=-\omega_{1}^{2} q_{1}-\int_{0}^{t} \gamma_{\mathrm{eq}}\left(t-t^{\prime}\right) \dot{q}_{1}\left(t^{\prime}\right) \mathrm{d} t^{\prime}+\xi_{\mathrm{eq}}(t),
$$

with the equilibrium friction kernel

$$
\begin{aligned}
\gamma_{\mathrm{eq}}(t) & =a^{2} \omega_{2}^{2} \exp \left(-\frac{\zeta}{2} t\right)\left[\cos (v t)-\frac{\zeta}{2 v} \sin (v t)\right], \\
\nu & \stackrel{\text { def }}{=}\left(\omega_{2}^{2}-\frac{\zeta^{2}}{4}\right)^{1 / 2} .
\end{aligned}
$$

Figure 3 shows the friction kernel $\gamma\left(t, t^{\prime}\right)$ as a function of the time difference $t-t^{\prime}$ for various values of the initial time $t^{\prime}$. Although the rough behavior (oscillation of frequency $\approx \omega_{2}$ and decay of $\approx \zeta / 2$ ) is similar for all $t^{\prime}$, the amplitude and the positions of the peaks and the zeros deviate appreciably with $t^{\prime}$. As time goes to infinity, the friction kernel also converges to the equilibrium one. Note that the equilibrium friction kernel $\gamma_{\mathrm{eq}}\left(t-t^{\prime}\right)$ becomes zero for $v\left(t-t^{\prime}\right)=\arctan (2 v / \zeta)+n \pi$ with $n$ being integers, that is, $t-t^{\prime}=0.76,2.33,3.91,5.48,7.05,8.63, \ldots$ If the 
nonstationary friction kernel were given by Eq. (10), it would be identically zero at these values of $t-t^{\prime}$ for any value of $t^{\prime}$. However, Fig. 3 shows that, for some $t^{\prime}, \gamma\left(t, t^{\prime}\right)$ is significantly different from zero even at these values of $t-t^{\prime}$. This implies that the friction kernel of this system does not fall into the category of Eq. (10).

Note also that the linear friction term in Eqs. (77) and (83) is an approximation. The exact formula is given by nonlinear friction term as in Eq. (64). It would be interesting in the future to analyze the simple example presented here or more realistic systems with the use of Eq. (64), and see whether the nonlinearity in the friction term changes due to the nonstationarity of the environment.

\section{SUMMARY AND OUTLOOK}

The generalized Langevin equation, which enables a lowdimensional description of a large system consisting of huge number of atoms including solvents, was extended to the case of nonstationary distribution of the bath. The derivation utilized the projection operator formalism, which starts from the original Hamiltonian of the total system without any assumption about the specific functional form of the Hamiltonian. The resulting form of iGLE is as simple enough as the usual GLE. The concept of the generalized friction kernel depending on both the past and the present was found to hold generally in any nonstationary system. The generalized fluctuationdissipation relation, ${ }^{28,29,34}$ found previously by heuristic arguments, was also proved to hold for any nonstationary system, when we interpret the temperature as the average of the square of the velocity of the system. In addition to the general and rigorous derivation that is free from any preassumption on the specific form of the underlying total Hamiltonian system, the other striking differences from the previous work are that the mean force appearing in the iGLE is time dependent, and the simple scaling relation ${ }^{28,29,34}$ of the random force and the friction kernel does not hold in general.

Recently, some attempts have been reported to evaluate the friction kernel from realistic molecular dynamics simulations, ${ }^{40-42}$ by which the manybody complex phenomena were successfully explained by combining with the GLEbased Grote-Hynes theory. ${ }^{2}$ Such analyses were, however, limited to equilibrium bath, since GLE is only for stationary systems. The present study provides us with a firm framework for the understanding of manybody complex phenomena occurring in a fluctuating environment, covering from equilibrium to nonequilibrium conditions with nonstationary bath. Some interesting subjects to be addressed in terms of the present theory are, for example, chemical reactions or structural change of biomolecules following some stimuli (such as light) not on the progress variable to describe the reaction or the structural change of the molecule but on the bath degrees of freedom which couple with the variable, resulting in a nonstationary environment. The other stimulating subject is the combination of the present theory and the recently developed dynamical reaction theory to extract the rigorous reaction coordinate to dominate the fate of reactions under thermal fluctuation in equilibrium. ${ }^{6-17}$ These should provide us with great new insights into many molecular events occurring in nonstationary environments.

\section{ACKNOWLEDGMENTS}

This work has been supported by Research Fellowships of the Japan Society for the Promotion of Science for Young Scientists (to S.K.) and by JSPS, JST/CREST, Grant-in-Aid for Research on Priority Areas "Molecular Theory for Real Systems" and "Innovative Nanoscience" (to T.K.). The computations were partially performed using Research Center for Computational Science, Okazaki, Japan.

\section{APPENDIX A: PROOF OF EQ. (47)}

Since $\left\{f_{j}(\boldsymbol{A})\right\}$ is a complete set of the functions of $\boldsymbol{A}$, its orthonormalization $\left\{\phi_{j, t}(\boldsymbol{A})\right\}$ is also a complete set. This means that any arbitrary function $g(\boldsymbol{A})$ of $\boldsymbol{A}$ can be expressed

$$
g(\boldsymbol{A})=\sum_{j} c_{j} \phi_{j, t}(\boldsymbol{A}),
$$

with the coefficients given by

$$
c_{j}=\left(\phi_{j, t} \mid g\right)_{t},
$$

from the orthonormality of $\left\{\phi_{j, t}(\boldsymbol{A})\right\}$. Therefore,

$$
\begin{aligned}
g(\boldsymbol{a})= & \sum_{j}\left(\phi_{j, t} \mid g\right)_{t} \phi_{j, t}(\boldsymbol{a}) \\
= & \sum_{j} \int \mathrm{d} \boldsymbol{q} \mathrm{d} \boldsymbol{p} \rho(\boldsymbol{q}, \boldsymbol{p}, t) \phi_{j, t}^{*}(\boldsymbol{A}) g(\boldsymbol{A}) \phi_{j, t}(\boldsymbol{a}), \\
= & \sum_{j} \int \mathrm{d} \boldsymbol{q} \mathrm{d} \boldsymbol{p} \rho(\boldsymbol{q}, \boldsymbol{p}, t) \int \mathrm{d} \boldsymbol{a}^{\prime} \delta\left(\boldsymbol{A}-\boldsymbol{a}^{\prime}\right) \phi_{j, t}^{*}\left(\boldsymbol{a}^{\prime}\right) g\left(\boldsymbol{a}^{\prime}\right) \\
& \phi_{j, t}(\boldsymbol{a}), \\
= & \sum_{j} \int \mathrm{d} \boldsymbol{a}^{\prime} P_{t}\left(\boldsymbol{a}^{\prime}\right) \phi_{j, t}^{*}\left(\boldsymbol{a}^{\prime}\right) \phi_{j, t}(\boldsymbol{a}) g\left(\boldsymbol{a}^{\prime}\right),
\end{aligned}
$$

where we have used the definition of $P_{t}$ in Eq. (48). Since this equation holds for any function $g$, we have

$$
\sum_{j} P_{t}\left(\boldsymbol{a}^{\prime}\right) \phi_{j, t}^{*}\left(\boldsymbol{a}^{\prime}\right) \phi_{j, t}(\boldsymbol{a})=\delta\left(\boldsymbol{a}-\boldsymbol{a}^{\prime}\right)
$$

\section{APPENDIX B: SOME IDENTITIES USED IN TEXT}

First we prove

$$
\hat{\mathscr{P}} f(\boldsymbol{A}, t)=f(\boldsymbol{A}, t),
$$

for any function $f$ of only $\boldsymbol{A}$ and $t$, and $\hat{\mathscr{P}}$ defined in Sec. III C. This is proved by

$$
\begin{aligned}
\hat{\mathscr{P}} f(\boldsymbol{A}, t)= & \sum_{j, n} \phi_{j, t}(\boldsymbol{A}) \chi_{n}(t) \int \mathrm{d} \boldsymbol{q}^{\prime} \mathrm{d} \boldsymbol{p}^{\prime} \mathrm{d} t^{\prime} \rho\left(\boldsymbol{q}^{\prime}, \boldsymbol{p}^{\prime}, t^{\prime}\right) \\
& \times \phi_{j, t}\left(\boldsymbol{A}\left(\boldsymbol{q}^{\prime}, \boldsymbol{p}^{\prime}\right)\right) \chi_{n}\left(t^{\prime}\right) f\left(\boldsymbol{A}\left(\boldsymbol{q}^{\prime}, \boldsymbol{p}^{\prime}\right), t^{\prime}\right),
\end{aligned}
$$




$$
\begin{aligned}
= & P_{t}(\boldsymbol{A})^{-1} \int \mathrm{d} \boldsymbol{q}^{\prime} \mathrm{d} \boldsymbol{p}^{\prime} \mathrm{d} t^{\prime} \delta\left(\boldsymbol{A}\left(\boldsymbol{q}^{\prime}, \boldsymbol{p}^{\prime}\right)-\boldsymbol{A}\right) \\
& \times \delta\left(t-t^{\prime}\right) f\left(\boldsymbol{A}\left(\boldsymbol{q}^{\prime}, \boldsymbol{p}^{\prime}\right), t^{\prime}\right) \rho\left(\boldsymbol{q}^{\prime}, \boldsymbol{p}^{\prime}, t^{\prime}\right) \\
= & P_{t}(\boldsymbol{A})^{-1} \int \mathrm{d} t^{\prime} P_{t^{\prime}}(\boldsymbol{A}) \delta\left(t-t^{\prime}\right) f\left(\boldsymbol{A}, t^{\prime}\right), \\
= & f(\boldsymbol{A}, t) .
\end{aligned}
$$

Next we prove

$$
\hat{\mathscr{P}}_{f}(\boldsymbol{A}, t) G=f(\boldsymbol{A}, t) \hat{\mathscr{P}}_{G},
$$

where $f$ is a function of only $\boldsymbol{A}$ and $t$, but $G$ is any function on the extended phase space. The proof proceeds similarly

$\hat{\mathscr{P}} f(\boldsymbol{A}, t) G$

$$
\begin{aligned}
= & \sum_{j, n} \phi_{j, t}(\boldsymbol{A}) \chi_{n}(t) \int \mathrm{d} \boldsymbol{q}^{\prime} \mathrm{d} \boldsymbol{p}^{\prime} \mathrm{d} t^{\prime} \rho\left(\boldsymbol{q}^{\prime}, \boldsymbol{p}^{\prime}, t^{\prime}\right) \\
& \times \phi_{j, t}\left(\boldsymbol{A}\left(\boldsymbol{q}^{\prime}, \boldsymbol{p}^{\prime}\right)\right) \chi_{n}\left(t^{\prime}\right) f\left(\boldsymbol{A}\left(\boldsymbol{q}^{\prime}, \boldsymbol{p}^{\prime}\right), t^{\prime}\right) G\left(\boldsymbol{q}^{\prime}, \boldsymbol{p}^{\prime}, t^{\prime}\right), \\
= & P_{t}(\boldsymbol{A})^{-1} \int \mathrm{d} \boldsymbol{q}^{\prime} \mathrm{d} \boldsymbol{p}^{\prime} \mathrm{d} t^{\prime} \delta\left(\boldsymbol{A}\left(\boldsymbol{q}^{\prime}, \boldsymbol{p}^{\prime}\right)-\boldsymbol{A}\right) \\
& \times \delta\left(t-t^{\prime}\right) f\left(\boldsymbol{A}\left(\boldsymbol{q}^{\prime}, \boldsymbol{p}^{\prime}\right), t^{\prime}\right) G\left(\boldsymbol{q}^{\prime}, \boldsymbol{p}^{\prime}, t^{\prime}\right) \rho\left(\boldsymbol{q}^{\prime}, \boldsymbol{p}^{\prime}, t^{\prime}\right), \\
= & f(\boldsymbol{A}, t) P_{t}(\boldsymbol{A})^{-1} \int \mathrm{d} \boldsymbol{q}^{\prime} \mathrm{d} \boldsymbol{p}^{\prime} \mathrm{d} t^{\prime} \delta\left(\boldsymbol{A}\left(\boldsymbol{q}^{\prime}, \boldsymbol{p}^{\prime}\right)-\boldsymbol{A}\right) \\
& \times \delta\left(t-t^{\prime}\right) G\left(\boldsymbol{q}^{\prime}, \boldsymbol{p}^{\prime}, t^{\prime}\right) \rho\left(\boldsymbol{q}^{\prime}, \boldsymbol{p}^{\prime}, t^{\prime}\right), \\
= & f(\boldsymbol{A}, t) \sum_{j, n} \phi_{j, t}(\boldsymbol{A}) \chi_{n}(t) \int \mathrm{d} \boldsymbol{q}^{\prime} \mathrm{d} \boldsymbol{p}^{\prime} \mathrm{d} t^{\prime} \rho\left(\boldsymbol{q}^{\prime}, \boldsymbol{p}^{\prime}, t^{\prime}\right) \\
& \times \phi_{j, t}\left(\boldsymbol{A}\left(\boldsymbol{q}^{\prime}, \boldsymbol{p}^{\prime}\right)\right) \chi_{n}\left(t^{\prime}\right) G\left(\boldsymbol{q}^{\prime}, \boldsymbol{p}^{\prime}, t^{\prime}\right) \\
= & f(\boldsymbol{A}, t) \hat{\mathscr{P}}_{G} .
\end{aligned}
$$

Another useful identity is

$$
\begin{aligned}
\exp ((1-\hat{\mathscr{P}}) \mathrm{i} \hat{\Lambda} \tau) f(t)(1-\hat{\mathscr{P}}) G \\
=f(t+\tau) \exp ((1-\hat{\mathscr{P}}) \mathrm{i} \hat{\Lambda} \tau)(1-\hat{\mathscr{P}}) G
\end{aligned}
$$

for any function $f$ of only $t$ and any function $G$ on the extended phase space. To prove this, take the differentiation of the right hand side:

$$
\begin{aligned}
\frac{\partial}{\partial \tau}[ & f(t+\tau) \exp ((1-\hat{\mathscr{P}}) \mathrm{i} \hat{\Lambda} \tau)(1-\hat{\mathscr{P}}) G] \\
& =f^{\prime}(t+\tau) \exp ((1-\hat{\mathscr{P}}) \mathrm{i} \hat{\Lambda} \tau)(1-\hat{\mathscr{P}}) G \\
& +f(t+\tau)(1-\hat{\mathscr{P}}) \mathrm{i} \hat{\Lambda} \exp ((1-\hat{\mathscr{P}}) \mathrm{i} \hat{\Lambda} \tau)(1-\hat{\mathscr{P}}) G .
\end{aligned}
$$

Since $\mathrm{i} \hat{\Lambda}$ is a first-order differential operator, and because of Eq. (B3),

$$
\begin{aligned}
&(1-\hat{\mathscr{P}}) \mathrm{i} \hat{\Lambda} f(t+\tau) \exp ((1-\hat{\mathscr{P}}) \mathrm{i} \hat{\Lambda} \tau)(1-\hat{\mathscr{P}}) G \\
&=(1-\hat{\mathscr{P}}) f^{\prime}(t+\tau) \exp ((1-\hat{\mathscr{P}}) \mathrm{i} \hat{\Lambda} \tau)(1-\hat{\mathscr{P}}) G \\
&+(1-\hat{\mathscr{P}}) f(t+\tau) \mathrm{i} \hat{\Lambda} \exp ((1-\hat{\mathscr{P}}) \mathrm{i} \hat{\Lambda} \tau)(1-\hat{\mathscr{P}}) G \\
&= f^{\prime}(t+\tau)(1-\hat{\mathscr{P}}) \exp ((1-\hat{\mathscr{P}}) \mathrm{i} \hat{\Lambda} \tau)(1-\hat{\mathscr{P}}) G \\
&+f(t+\tau)(1-\hat{\mathscr{P}}) \mathrm{i} \hat{\Lambda} \exp ((1-\hat{\mathscr{P}}) \mathrm{i} \hat{\Lambda} \tau)(1-\hat{\mathscr{P}}) G \\
&= f^{\prime}(t+\tau) \exp ((1-\hat{\mathscr{P}}) \mathrm{i} \hat{\Lambda} \tau)(1-\hat{\mathscr{P}}) G \\
&+f(t+\tau)(1-\hat{\mathscr{P}}) \mathrm{i} \hat{\Lambda} \exp ((1-\hat{\mathscr{P}}) \mathrm{i} \hat{\Lambda} \tau)(1-\hat{\mathscr{P}}) G .
\end{aligned}
$$

Equation (B6) therefore becomes

$$
\begin{aligned}
& \frac{\partial}{\partial \tau}[f(t+\tau) \exp ((1-\hat{\mathscr{P}}) \mathrm{i} \hat{\Lambda} \tau)(1-\hat{\mathscr{P}}) G] \\
= & (1-\hat{\mathscr{P}}) \mathrm{i} \hat{\Lambda} f(t+\tau) \exp ((1-\hat{\mathscr{P}}) \mathrm{i} \hat{\Lambda} \tau)(1-\hat{\mathscr{P}}) G .
\end{aligned}
$$

Thus both sides of Eq. (B5) is a solution of the differential equation

$$
\frac{\partial}{\partial \tau} \Psi=(1-\hat{\mathscr{P}}) \mathrm{i} \hat{\Lambda} \Psi
$$

and they are equal at $\tau=0$. Therefore the two sides of Eq. (B5) are equal for all $\tau$, because of the uniqueness of the solution of the differential equation Eq. (B9).

\section{APPENDIX C: PROOF OF EQ. (68)}

We will show here

$$
\left\langle\tilde{\xi}_{j}(\tau-s) \xi(\tau)\right\rangle_{0}=\left\langle\tilde{\xi}_{j}(0) \xi(s)\right\rangle_{\tau-s}
$$

Then, since the distribution does not change in the stationary state, we have

$$
\left\langle\tilde{\xi}_{j}(0) \xi(s)\right\rangle_{\tau-s} \stackrel{\text { stationary }}{=}\left\langle\tilde{\xi}_{j}(0) \xi(s)\right\rangle_{0} .
$$

The proof of Eq. (C1) is as follows: 


$$
\begin{aligned}
\left\langle\tilde{\xi}_{j}(\tau-s) \boldsymbol{\xi}(\tau)\right\rangle_{0}, & =\sum_{n} \chi_{n}(0)\left(\left(\chi_{n}(t) \tilde{\xi}_{j}(\tau-s) \mid \boldsymbol{\xi}(\tau)\right)\right) \\
& =\sum_{n} \chi_{n}(0)\left(\left(\chi_{n}(t) \exp ((1-\hat{\mathscr{P}}) \mathrm{i} \hat{\Lambda}(\tau-s)) \tilde{\xi}_{j}(0) \mid \boldsymbol{\xi}(\tau)\right)\right), \\
& =\sum_{n} \chi_{n}(0)\left(\left(\exp ((1-\hat{\mathscr{P}}) \mathrm{i} \hat{\Lambda}(\tau-s)) \chi_{n}(t-\tau+s) \tilde{\xi}_{j}(0) \mid \boldsymbol{\xi}(\tau)\right)\right), \\
& =\sum_{n} \chi_{n}(0)\left(\left(\chi_{n}(t-\tau+s) \tilde{\xi}_{j}(0) \mid \exp ((1-\hat{\mathscr{P}}) \mathrm{i} \hat{\Lambda}(s-\tau)) \boldsymbol{\xi}(\tau)\right)\right), \\
& =\sum_{n} \chi_{n}(0)\left(\left(\chi_{n}(t-\tau+s) \tilde{\xi}_{j}(0) \mid \boldsymbol{\xi}(s)\right)\right), \\
& =\left\langle\tilde{\xi}_{j}(0) \boldsymbol{\xi}(s)\right\rangle_{\tau-s},
\end{aligned}
$$

where the similar technique is used as Eqs. (58)-(62).

${ }^{1}$ H. A. Kramers, Physica 7, 284 (1940).

${ }^{2}$ R. F. Grote and J. T. Hynes, J. Chem. Phys. 73, 2715 (1980).

${ }^{3}$ R. Zwanzig, Nonequilibrium Statistical Mechanics (Oxford University Press, London, 2001).

${ }^{4}$ H. Mori, Prog. Theor. Phys. 33, 423 (1965).

${ }^{5}$ K. Kawasaki, J. Phys. A 6, 1289 (1973).

${ }^{6}$ C. C. Martens, J. Chem. Phys. 116, 2516 (2002).

${ }^{7}$ T. Bartsch, R. Hernandez, and T. Uzer, Phys. Rev. Lett. 95, 058301 (2005).

${ }^{8}$ T. Bartsch, T. Uzer, and R. Hernandez, J. Chem. Phys. 123, 204102 (2005).

${ }^{9}$ T. Bartsch, T. Uzer, J. M. Moix, and R. Hernandez, J. Chem. Phys. 124, 244310 (2006)

${ }^{10}$ T. Bartsch, J. M. Moix, R. Hernandez, S. Kawai, and T. Uzer, Adv. Chem. Phys. 140, 191 (2008).

${ }^{11}$ T. Bartsch, J. Chem. Phys. 131, 124121 (2009).

${ }^{12}$ R. Hernandez, T. Uzer, and T. Bartsch, Chem. Phys. 370, 270 (2010).

${ }^{13}$ S. Kawai and T. Komatsuzaki, J. Chem. Phys. 131, 224505 (2009).

${ }^{14}$ S. Kawai and T. Komatsuzaki, J. Chem. Phys. 131, 224506 (2009).

${ }^{15}$ S. Kawai and T. Komatsuzaki, Phys. Chem. Chem. Phys. 12, 7626 (2010).

${ }^{16}$ S. Kawai and T. Komatsuzaki, Phys. Chem. Chem. Phys. 12, 7636 (2010).

${ }^{17}$ S. Kawai and T. Komatsuzaki, Phys. Chem. Chem. Phys. 12, 15382 (2010).

${ }^{18}$ H. Sumi and R. A. Marcus, J. Chem. Phys. 84, 4894 (1986).

${ }^{19}$ E. Pollak, H. Grabert, and P. Hänggi, J. Chem. Phys. 91, 4073 (1989).

${ }^{20}$ A. M. Berezhkovskii, A. M. Frishman, and E. Pollak, J. Chem. Phys. 101, 4778 (1994)

${ }^{21}$ A. M. Berezhkovskii, V. Y. Zitserman, D. Y. Yang, J. Kuo, and S. H. Lin, Physica A 251, 399 (1998).
${ }^{22}$ B. C. Dian, A. Longarte, and T. S. Zwier, Science 28, 2369 (2002).

${ }^{23}$ K. Nakai, H. Kono, Y. Sato, N. Niitsu, R. Sahnoun, M. Tanaka, and Y. Fujimura, Chem. Phys. 338, 127 (2007).

${ }^{24}$ K. Iwata, J. Raman Spectrosc. 39, 1512 (2008).

${ }^{25}$ M. M. Millonas and C. Ray, Phys. Rev. Lett. 75, 1110 (1995).

${ }^{26}$ S. Bhattacharya, S. K. Banik, S. Chattopadhyay, and J. R. Chaudhuri, J. Math. Phys. 49, 063302 (2008).

${ }^{27}$ J. R. Chaudhuri, G. Gangopadhyay, and D. S. Ray, J. Chem. Phys. 109, 5565 (1998).

${ }^{28}$ R. Hernandez and F. L. Somer, Jr., J. Phys. Chem. B 103, 1064 (1999).

${ }^{29}$ R. Hernandez, J. Chem. Phys. 111, 7701 (1999).

${ }^{30}$ R. Hernandez and F. L. Somer, J. Phys. Chem. B 103, 1070 (1999).

${ }^{31}$ F. L. Somer and R. Hernandez, J. Phys. Chem. A 103, 11004 (1999).

${ }^{32}$ F. L. Somer and R. Hernandez, J. Phys. Chem. B 104, 3456 (2000).

${ }^{33}$ M. Vogt and R. Hernandez, J. Chem. Phys. 123, 144109 (2005).

${ }^{34}$ A. V. Popov and R. Hernandez, J. Chem. Phys. 126, 244506 (2007).

${ }^{35}$ R. Zwanzig, J. Stat. Phys. 9, 215 (1973).

${ }^{36}$ A. Lichtenberg and M. Lieberman, Regular and Chaotic Dynamics (Springer, New York, 1992).

${ }^{37}$ T. Komatsuzaki and R. S. Berry, Adv. Chem. Phys. 123, 79 (2002).

${ }^{38}$ M. Abramowitz and I. A. Stegun, Handbook of Mathematical Functions (Dover publications, New York, 1965).

${ }^{39}$ See supplementary material at http://dx.doi.org/10.1063/1.3561065 for the details of numerical calculation of the projection operator, the mean force, and the friction kernels.

${ }^{40}$ J. J. Ruiz-Pernía, I. Tuñón, V. Moliner, J. T. Hynes, and M. Roca, J. Am. Chem. Soc. 130, 7477 (2008).

${ }^{41}$ R. P. McRae, G. K. Schenter, B. C. Garrett, Z. Svetlicic, and D. G. Truhlar, J. Chem. Phys. 115, 8460 (2001).

${ }^{42}$ I. S. Tolokh, G. W.N. White, S. Goldman, and C. G. Gray, Mol. Phys. 100, 2351 (2002). 